\title{
Ultra-Low Cost Vehicle Data Acquisition and Transfer System from Analog and Digital Sensors to Audio Channel of a Phone
}

\author{
Ajinkya Bari ${ }^{\mathrm{a}}$, Shraddhesh Bhandari ${ }^{\mathrm{a}}$, Rajesh Krishnan ${ }^{\mathrm{b}}$, Rohit Pandharkar ${ }^{\mathrm{c}}$ \\ ${ }^{a}$ Department of Electronics \& Telecommunication Engineering, COEP (College of Engineering, Pune), India \\ ${ }^{b}$ Department of Instrumentation \& Control Engineering, COEP (College of Engineering, Pune), India \\ ${ }^{c}$ Mahindra \& Mahindra Ltd., India \\ barias10.extc@coep.ac.in, bhandarisv10.extc@coep.ac.in, rajeshk11.instru@coep.ac.in, \\ pandharkar.rohit@mahindra.com
}

\begin{abstract}
The proposed system acquires and transfers data from a vehicle's analog and digital sensors to the user's very own mobile phone. The device uses a microcontroller to accept the sensor inputs and generate an audio signal indicative of the data acquired by the sensors and an audio jack which on inserting into the phone acts as a channel to transfer the data collected by the sensors to the cell phone. This data acquired from the sensors is fed to the microphone jack of the cell phone which is then processed by a mobile application and decoded sensor values are displayed on the cell phone. The ultra-low cost nature of the technology enables new additional applications like: On-TheSpot Soil Testing, Home Automation, Traffic Data Capture and Health Data Capture, etc. at disruptive prices.
\end{abstract}

Keywords - Data Acquisition, Digital Signal processing, Fast Fourier Transform, Spatio-Frequency Encoding, Time Division Multiplexing, 3.5 mm Audio jack

\section{INTRODUCTION}

Vehicle data acquisition is a crucial part of automotive sensing techniques. In order to achieve this, various approaches have been proposed. Technologies like USB [1], Bluetooth [2], Wi-Fi [3] and NFC [4] have been extensively used to facilitate intra-vehicular communication. However, these technologies are differently implemented in different phones which hamper their compatibility to a great extent. Additionally, these technologies also require substantial hardware which increases the cost, complexity and power consumption of the entire system. To enable vehicular data acquisition systems in 2wheelers and low end commercial vehicles like 3-wheelers in emerging countries, we propose a technology to achieve the same at disruptive costs. Our system uses analog signals in the audible frequency range to establish communication between various devices and the user's very own mobile phone. Every mobile phone recognizes the audible range of sound signals which makes it a cogent choice for our application. This technology finds its applications in various domains -

- Automobiles - Connected Car [5], [6] Technology

- Medical - Health Data Capture and Transfer

- Agriculture - On The Spot Soil Testing

- Domestic - Home Automation

\section{- Infrastructure - Traffic Data Capture}

However, in this paper we focus on vehicular communication systems. Considering the example of Connected Cars, one can observe that installing a cellular GPS system on a vehicle is both complex and cost intensive. On the other hand if we read the data in a mobile phone, we can use the pre-existing GPS/ GPRS technologies present in it, thereby reducing costs drastically. The SIM card of the mobile phone can also be used to communicate with servers and satellites. On the cost side, it is important to note that the cost of the device (GPS, SIM, Battery etc.) gets entirely shifted to the user's own cell phone having all the aforementioned features thus eliminating the need for additional circuitry making our system more resourceful and an economically apt choice.

We leverage the fact that worldwide mobile phone penetration is increasing by the day. Thus reusing the resources present in the existing smartphones and their platforms (Android, $3 \mathrm{G}$ etc.) reduces the complexity of data processing and transmission.

\section{PRIOR ART}

Prevailing systems use wireless and near field communication to acquire vehicle data and transmit it to a mobile phone. Transceivers are provided which are configured to communicate wirelessly within the vehicle with a cell phone using Bluetooth communication protocol as adopted in the said system [7]. Radio frequency transmission technology has been put into use in existing systems [8], [9] which transmit internal (subsystems, ambient temperature etc.) or external (roadway, atmosphere, etc.) vehicle data to a vehicle operator or an external site.

The system cited in [10] uses a vehicle portable device to acquire the vehicle information which is transmitted from the vehicle by wireless communication. It makes use of a transmission controller that controls the transmission of the vehicle information to the mobile terminal by near-field wireless communication. While the above mentioned systems use sensors to acquire vehicle information, yet another system 
[11] uses a camera which when pointed to a vehicle transmits information through images to a mobile computing device which is preferably a mobile phone having at least a camera, a display, a processor, and a tangible non-transient computerreadable medium for storing appropriate programming and vehicle information.

Square Reader [12] is a small plastic device that comprises of a read head for passing a magnetic stripe of a card to read the stored data and produce an analog signal indicative of it which is provided to a cell phone through an output jack. The signal containing the magnetic strip data is transmitted to the phone microprocessor which amplifies, samples and decodes the data with the aid of a pre-installed application. However, in our system the acquired sensor data is Spatio-Frequency encoded leveraging properties of sound waves and transmitted in the form of audio signals to the cell phone.

\section{SPATIO-FREQUENCY ENCODING}

We have used Spatio-Frequency encoding to transfer the sensor values to the phone. In this, the sensor output is SpatioFrequency encoded leveraging properties of audio signals.

The output of the sensor is converted to an audio signal as shown in (1).

$$
Y(t)=C \sin \left(2 \pi f_{t} t\right)
$$

In this conversion, the signal from the sensor undergoes spatial encoding as the sensor output is converted into a continuous audio signal with frequency indicative of the output of the sensor as shown in Figure 1(a). Eq. (2) shows the Fourier Transform (FT) [13] of audio signal in (1).

$$
Y(X)=F T(Y(t))
$$

The signal $Y(X)$ is now frequency encoded in which the ideal frequency response $(H(X))$ of the phone microphone is normalized using an inverse frequency response $\left(H^{\prime}(X)\right)$ to achieve a constant flat filter response (for maintaining constant SNR) in the desired frequency range as shown in Figure 1(b). Thus the sensor output is first encoded in the spatial domain and then in the frequency domain to obtain optimum results for our system.

The resultant Spatio-Frequency encoded signal $\left(Y^{\prime}(t)\right)$ is given by

$$
Y^{\prime}(t)=\operatorname{IFT}\left(Y(X) * H^{\prime}(X)\right),
$$

where IFT denotes Inverse Fourier Transform.

\section{PROPOSED APPROACH}

\section{A. Algorithmic Methodology}

As shown in Table I. consider the output of the sensor to be

\section{Spatial Encoding}

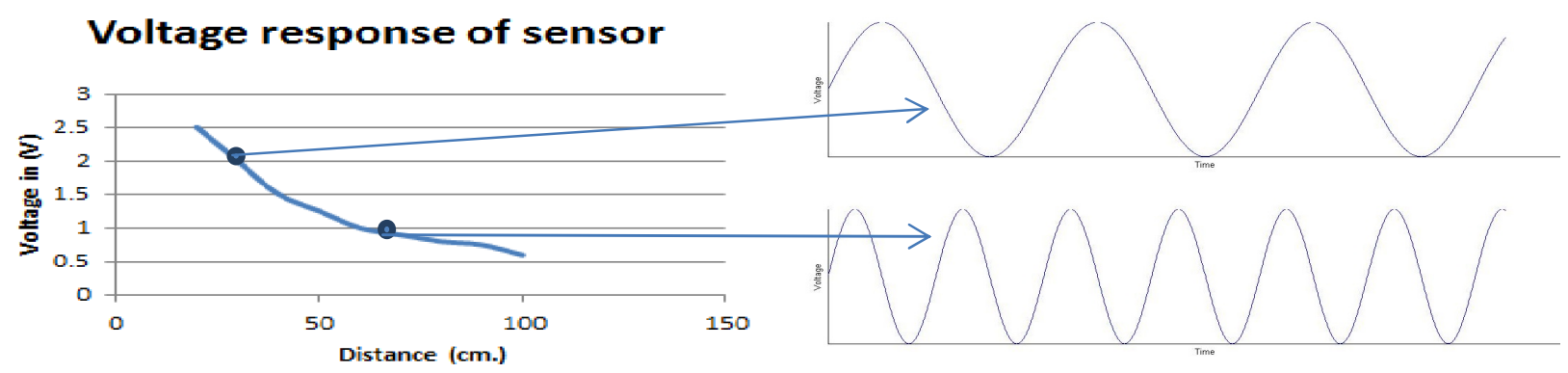

(a) Spatial Encoding: The sensor values are converted to audio signals having different frequencies

\section{Frequency Encoding}

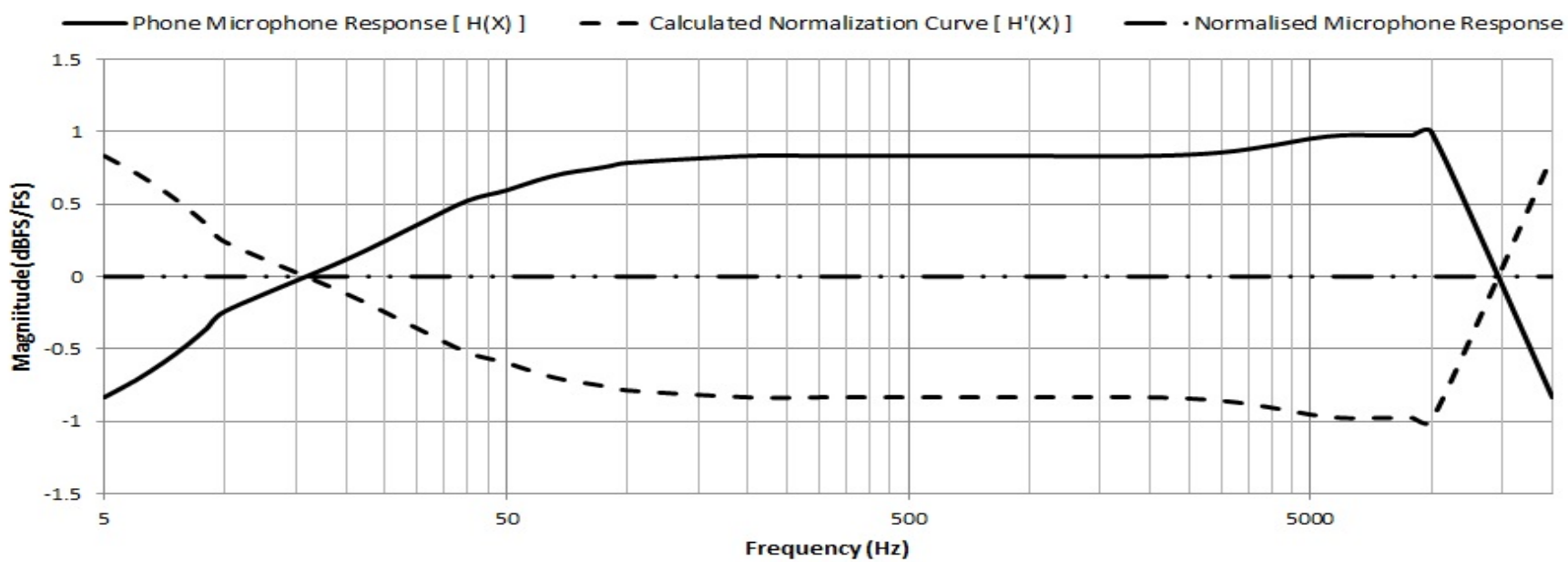

(b) Frequency Encoding: This shows the normalization of the phone microphone frequency response

Figure 1. Spatio- Frequency Encoding 


$$
S(t)=g(D) .
$$

This signal is given to the ADC of the microcontroller which converts it into 12 bit digital samples. Therefore the digital value $S_{A D C}$ can be represented as

$$
S_{A D C}=\left(\frac{s(t)}{V_{\text {ref }}}\right) * 2^{12},
$$

where $V_{r e f}=$ Reference voltage of the ADC.

Depending on this value acquired from the sensor, a sine wave with proportional frequency in the audible range of sound is generated from the microcontroller as seen in (1). This signal after Spatio-Frequency encoding is routed through the audio cable to the mobile phone via the $3.5 \mathrm{~mm}$ audio jack like any other sound wave. The signal received by the phone microprocessor is then sampled [14] at a frequency of 44100 $\mathrm{Hz}$. The sampled signal can be represented as

$$
Y(n)=C \sin \left(2 \pi\left(\frac{f_{t}}{f_{s}}\right) n\right)
$$

These digital samples are used to calculate the frequency of the signal using the Cooley-Tukey FFT [15] algorithm. Once the frequency is computed, linear regression is used to map it to the value of the distance of the object from the sensor which is then displayed on the screen.

For interfacing multiple sensors simultaneously we have used the concept of Time Division Multiplexing in which the signal corresponding to each sensor is sent to the phone for a predefined time interval. Consider $N$ sensors $\mathrm{S}_{1}, \mathrm{~S}_{2}, \mathrm{~S}_{3}$, up to $\mathrm{S}_{\mathrm{N}}$ having outputs $S_{1}(t), S_{2}(t)$ up to $S_{N}(t)$ respectively. Each of the sensor output is sent to different ADC channels of the microcontroller. These values are then converted to corresponding digital values by the ADC. These digital values are used to generate sine waves with frequency proportional to the amplitude of the sensor outputs. In order to distinguish different sensors, a predefined frequency is attached at the beginning of each sine wave analogous to the start bit in digital data transmission. Thus a continuous train of audio signals is sent to the microprocessor of the phone with different sensor data interleaved between them. The frequency of the received audio signal is computed in the phone and the corresponding sensor data is calculated using regression equations and displayed on the screen.

As the number of sensors $N$, the initial phase of the signal $\varphi$, the reference voltage for the ADC module $V_{\text {ref }}$ and the sampling frequency in the phone $f_{s}$ remain fixed, the system becomes universal and can be used with any cell phone.

\section{B. Hardware Used}

1) Microcontroller: For experimental purposes, we have used the MSP 430FG4618 [16] microcontroller manufactured by Texas Instruments. It is a 16-bit mixed signal RISC microcontroller which is capable of handling both analog and digital signals thereby increasing the scope of our experiment.

2) $3.5 \mathrm{~mm}$ Audio Jack: The $3.5 \mathrm{~mm}$ jack having the TRRS (Tip Ring Ring Sleeve) topography has 4 contacts: Tip (Left speaker), Ring (Right speaker), Ring (Microphone), Sleeve (Ground) separated by insulators. The audio jack is capable of accepting analog audio signals through the microphone which can then be processed by the phone.

TABLE I. SYSTEM OVERVIEW

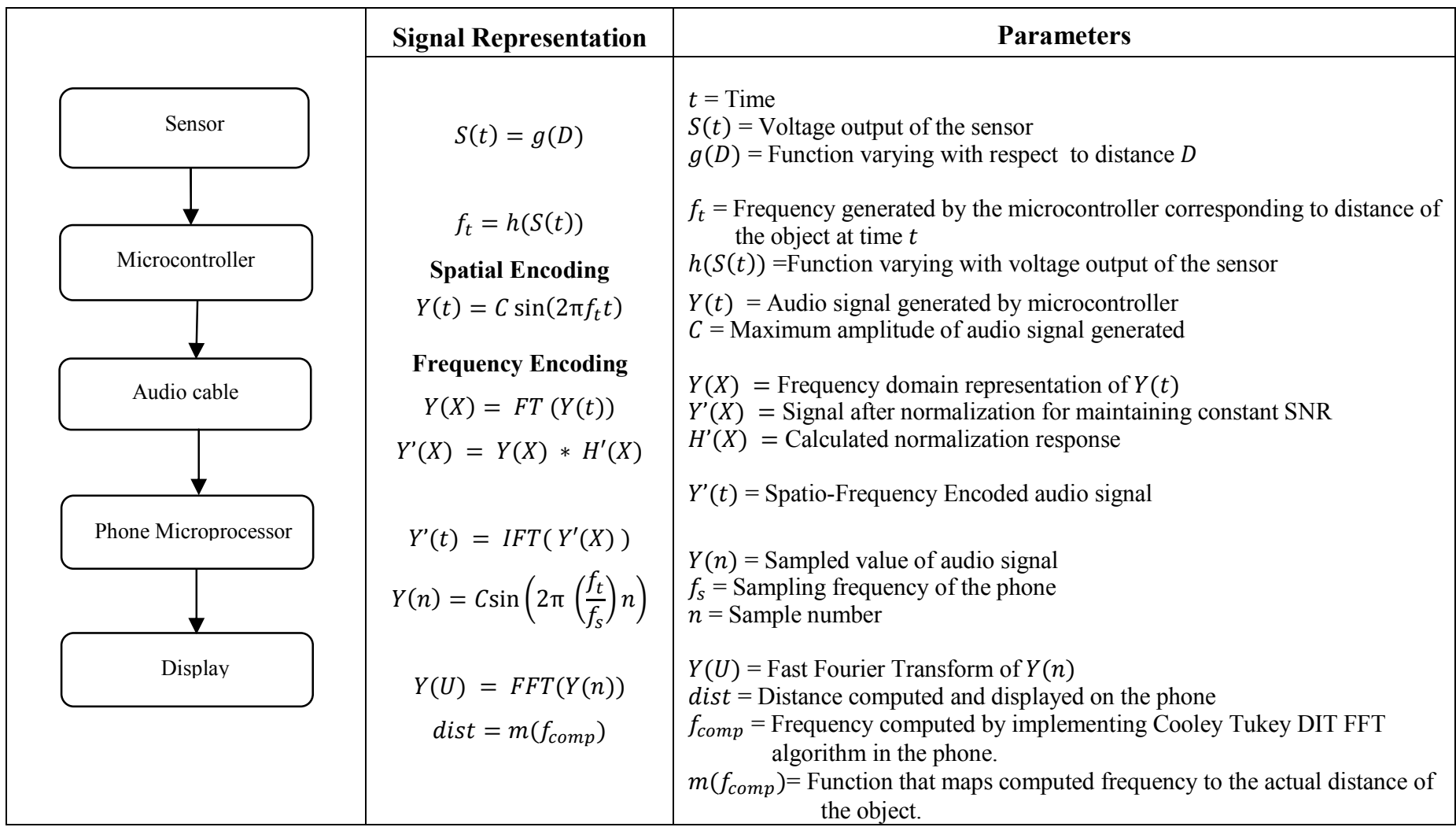


3) Low Pass Filter: A simple $1^{\text {st }}$ order R-C low pass filter with cut off frequency $6 \mathrm{KHz}$ has been used to smoothen the frequency modulated waves outputted by the DAC of the microcontroller.

Resistor Value Used $-5.6 \mathrm{k} \Omega$

Capacitor Value Used $-4.7 \mathrm{nF}$

4) Infrared (IR) Sensor: During the developmental phase, we used an analog infrared distance sensor SHARP GP2Y0A02YK [17]. An object within $1 \mathrm{~m}$ range can be accurately detected by the sensor. The output voltage ranges from $0.5 \mathrm{~V}$ to $2.5 \mathrm{~V}$ depending on the distance of the object from the sensor.

5) Low Cost Android Phone: A low cost Android [18] phone has been used to process and display the incoming information. The device is loaded with Ice Cream Sandwich $\mathrm{OS}$ and can accept input signals ranging from $-1.5 \mathrm{~V}$ to +1.5 $\mathrm{V}$. The input signals fed from the audio jack are sampled at $44.1 \mathrm{Khz}$. The overall system overview is summarized in Figure 2.

\section{Software}

The microcontroller is programmed to continuously generate a sine wave using its DAC Module. In order to generate a sine wave, we have used a simple look-up table approach. In this, the DAC module register is continuously updated with values that represent a sampled sine wave in the overflow interrupt service routine of a 16-bit Timer (Timer B) operated in the UP mode. In this mode, the timer counts up to a specified count and when the count is reached an interrupt is generated. Thus by varying the count of the Timer overflow, the frequency of the sine wave can be varied. The working of the microcontroller is summarised in Figure 3. On powering ON, the microcontroller accepts the sensor value and converts it into digital data using the ADC module. The Timer compare register is then loaded with a count indicative of the ADC value so as to vary the frequency of the sine wave according to the value of the sensor.

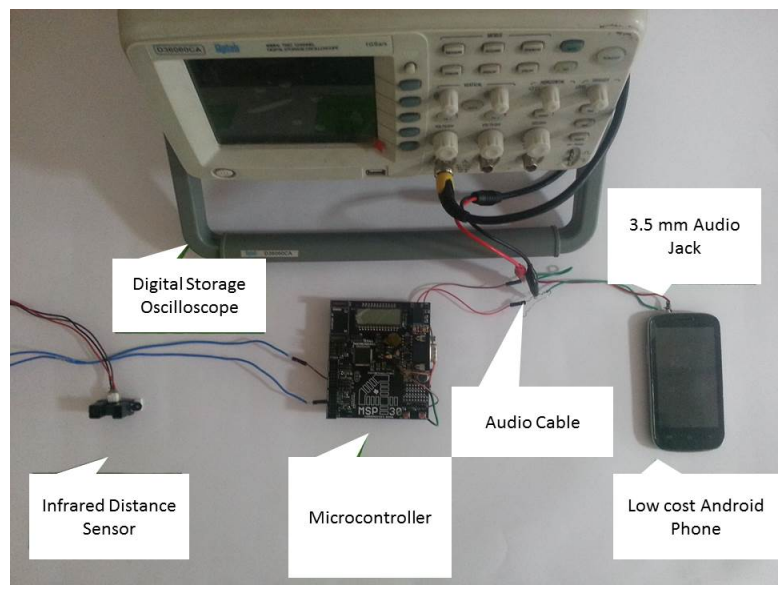

Figure 2. Overall System Overview

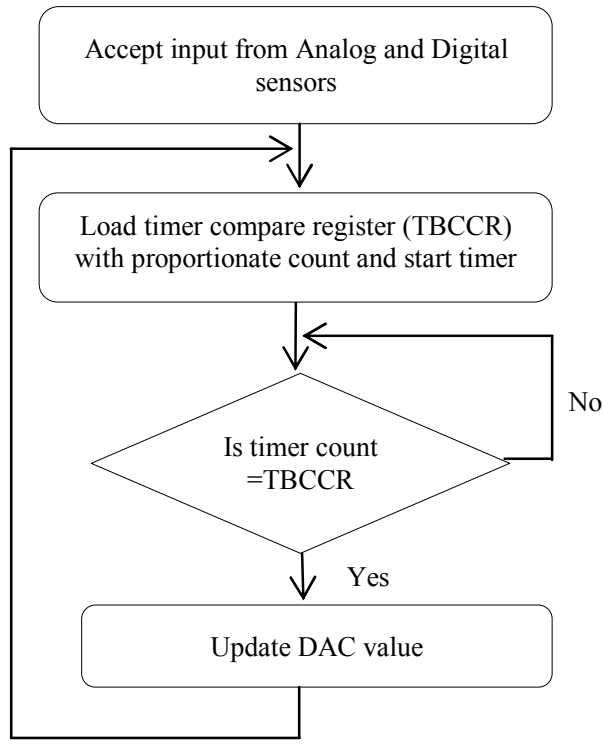

Figure 3. Algorithm for generation of audio signals corresponding to sensor output using microcontroller

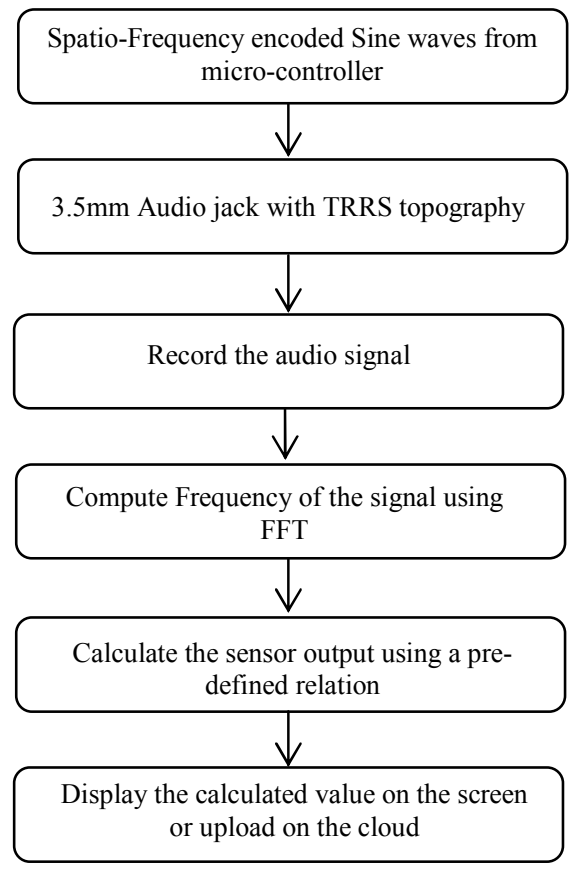

Figure 4. Algorithm for decoding frequency of the signal in cell phone

Figure 4 summarizes the processing of the audio signal in the mobile phone. The Android application reads the data from the audio buffer of the phone. This data is nothing but the sampled values of the incoming signal at the audio jack. Most commonly used sampling rate in mobile phones is $44100 \mathrm{~Hz}$ and the maximum buffer size that can be read at once is 4096 bytes and hence the same values have been used. The audio buffer containing the sampled values is read continuously in a separate thread. The Cooley-Tukey FFT algorithm is used to compute the frequencies from these sampled values. 


\section{EXPERIMENTATION}

\section{A. Experimental Setup}

For experimental and illustrative purposes, we have modeled the rear obstacle distance measuring systems in cars using an infrared distance sensor. Our system takes the distance data captured in voltage by the infrared sensor and transmits it to the cell phone through the audio channel. This transmission is Spatio-Frequency encoded. The phone decodes back the Spatio-Frequency encoded data into intelligible information and displays it to the user.

Figure 5 shows the experimental setup of the system during research. Both the microcontroller board and the sensor are powered using a $9 \mathrm{~V}$ battery. We have used a DC voltage regulator IC 7805 to step down $9 \mathrm{~V}$ to $5 \mathrm{~V}$. The infrared sensor output is given to the ADC0 pin of the microcontroller. The DAC0 pin output from the microcontroller is given to a low pass filter, the output of which is given to the audio jack of the cell phone.

\section{B. Calibration}

1) Distance to Voltage: Initially we calibrated the voltage level outputted by the sensor to the distance of the object from the sensor. The output of the sensor varies with the distance of the object with a voltage of $0.5 \mathrm{~V}$ for a distance of $100 \mathrm{~cm}$ and $2.5 \mathrm{~V}$ for a distance of $20 \mathrm{~cm}$

2) Volatge to Frequency: The acquired ADC value is mapped to the frequency generated by the microcontroller in this stage using the equation

$$
T B C R R 0=100+\frac{S_{A D C}}{20},
$$

where TBCRR0 is the compare match count

We observed that the variation in the frequency was approximately linear with $2.654 \mathrm{KHz}$ corresponding to $2.5 \mathrm{~V}$ and $5.205 \mathrm{KHz}$ corresponding to $0.5 \mathrm{~V}$.

3) Frequency to Distance: Quadratic regression was used to accurately map the computed frequency $f$ to the corresponding distance $d$ as data points fit the second order curve with comparatively minute error. We have used the following two regression equations:

$$
d=4.2406 f^{2}-5.4861 f+4.5536 \quad f \leq 5 \mathrm{KHz}
$$

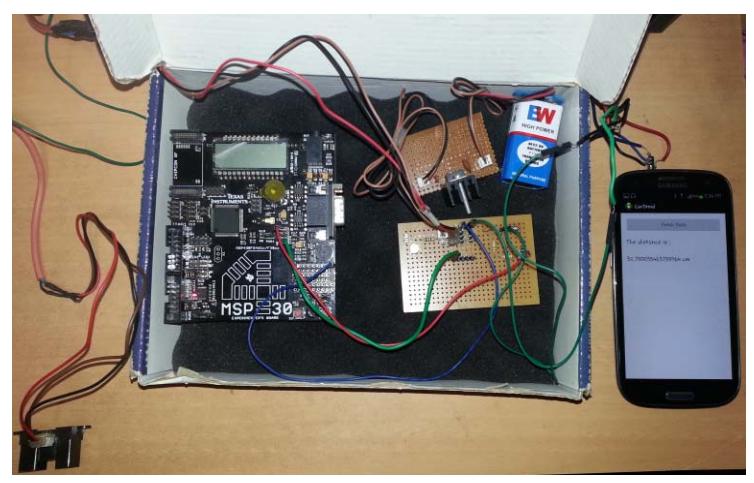

Figure 5. Experimental Setup

Above setup presents a system modeling the rear parking assist in cars using an IR distance sensor

$$
d=21.901 f^{2}-120.42 f+134.550 \quad f>5 K H z
$$

\section{Phone Application:}

Figure 6 shows a screenshot of a prototype application developed for an android cell phone. Two infrared distance sensors were interfaced with the system and using time division multiplexing technique, information was transmitted to the cell phone and the corresponding distances were computed.

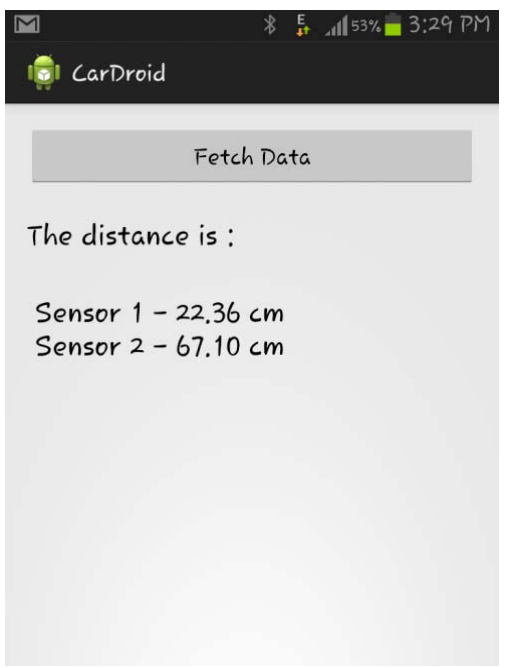

Figure 6. Android Application Screenshot Above screenshot displays the distance of two objects from corresponding sensors interfaced using Time Division Multiplexing

D. Observations:

TABLE II. EXPERIMENTAL OBSERVATIONS

\begin{tabular}{|c|c|c|c|c|}
\hline $\begin{array}{c}\text { Distance } \\
\text { of the } \\
\text { Object } \\
\text { (cm.) }\end{array}$ & $\begin{array}{c}\text { Frequency } \\
\text { Generated } \\
\text { by the } \\
\text { Controller } \\
\text { (Hz) }\end{array}$ & $\begin{array}{c}\text { Frequency } \\
\text { Calculated } \\
\text { in the } \\
\text { Phone } \\
\text { (Hz) }\end{array}$ & $\begin{array}{c}\text { Calculated } \\
\text { Distance } \\
\text { in the } \\
\text { phone } \\
\text { (cm.) }\end{array}$ & $\begin{array}{c}\text { Error } \\
\text { Percentage } \\
\text { (\%) }\end{array}$ \\
\hline 20 & 2654 & 2680.87 & 20.32 & 1.60 \\
\hline 25 & 2963 & 3003.46 & 26.33 & 5.32 \\
\hline 30 & 3247 & 3305.34 & 32.75 & 9.16 \\
\hline 35 & 3412 & 3445.31 & 35.98 & 2.80 \\
\hline 40 & 3623 & 3639.10 & 40.74 & 1.85 \\
\hline 45 & 3794 & 3822.14 & 45.53 & 1.17 \\
\hline 50 & 3991 & 4015.94 & 50.91 & 1.82 \\
\hline 55 & 4196 & 4242.04 & 57.59 & 4.71 \\
\hline 60 & 4303 & 4328.17 & 60.24 & 0.40 \\
\hline 65 & 4505 & 4532.73 & 66.81 & 2.78 \\
\hline 70 & 4668 & 4694.23 & 72.24 & 3.20 \\
\hline 75 & 4783 & 4812.67 & 76.37 & 1.83 \\
\hline 80 & 4993 & 4958.99 & 81.63 & 2.04 \\
\hline 85 & 5047 & 5049.03 & 84.86 & 0.16 \\
\hline 90 & 5107 & 5102.87 & 90.34 & 0.38 \\
\hline 95 & 5158 & 5156.71 & 95.96 & 1.01 \\
\hline 100 & 5205 & 5199.7 & 100.54 & 0.54 \\
\hline
\end{tabular}




\section{DISCUSSION OF RESULTS}

Table II shows the values of different parameters measured during experimentation. Computed distance values were found to be approximately conforming to the actual distance values with an average error of $2.37 \%$. The error can be minimized further by using highly sensitive sensors. The results show that our system can be effectively used to interface multiple sensors to the cell phone with the error being below the acceptable tolerance level.

\section{MERITS}

- The crucial advantage of this technique of data transfer is the use of $3.5 \mathrm{~mm}$ jack which makes the system completely universal and can be implemented using any mobile phone making it very versatile.

- The ultra-low cost nature of this system allows it to be also implemented in low end commercial vehicles in emerging countries.

- With the availability of data on a mobile phone, further improvisations can be made to the application by developing an application that communicates with a centralized server or cloud and optimizes data storage and processing.

- Although in our implementation, an infrared distance sensor was used, the ability to convert analog and digital output of any sensor to a Spatio-Frequency encoded wave allows the use of any sensor or transducer enabling the user to implement a variety of systems.

\section{DEMERITS}

- One of the limitations of our system is that it can only process speech signals thus limiting the overall system bandwidth.

- The accuracy and precision of the system is compromised as the number of sensors increase. This limits the maximum number of sensors that can be interfaced with the system.

\section{CONCLUSIONS}

We have successfully developed an ultra-low cost system to interface any type of sensor to a cell phone. Secondly, our system is scalable to any number of consumers. Furthermore, using voltage divider and signal conditioning circuits, sensors of various ratings can be interfaced with our system thus making it universal.

To conclude, the system we designed provides an economical alternative to other data transfer methodologies within vehicles and drastically reduces system complexity and costs. Our system can be additionally extended for many other diverse applications like Home Automation, Medical Diagnosis, Soil Testing, etc.

\section{ACKNOWLEDGMENT}

We would like to thank Mahindra \& Mahindra Ltd. for providing the necessary hardware and facilities required during the development of the system.

\section{REFERENCES}

[1] Universal Serial Bus, USB 2.0, 2000

[2] Bluetooth, IEEE 802.15.1, 2005

[3] WiFi, IEEE 802.11n, 2009

[4] Near Field Communication, ISO 13157-2, 2010

[5] Audi, Audi Connect Services, Product information: http://www.audi.com/com/brand/en/models/infotainment_and_communi cation/audi_connect_services.html

[6] BMW, BMW Connected, Product information:

http://www.bmw.com/com/en/insights/technology/connecteddrive/2013/ connecteddrive_services/index.html

[7] Communication system for vehicle, by Todd Witkowski, Kurt Dykema, Steven Geerlings, Mark Zeinstra, Robert Buege (2005, April 28). Patent US20050090279 A9 [Online]

Available https://www.google.com/patents/US20050090279

[8] Vehicle wireless sensing and communication system, by David $\mathrm{S}$ Breed, Wendell C. Johnson, Vittorio Castelli, William E. Seitz, Wilbur E. DuVall, (2003, December 16). Patent US 6662642 B2 [Online]

Availabe http://www.google.co.in/patents/US6662642

[9] System for obtaining vehicular information, by David S. Breed, Wilbur E. DuVall, Wendell C. Johnson (2008, September 2). Patent US $7421321 \mathrm{~B} 2$

Available:http://www.google.com/patents/US7421321?dq=US+742132 $1+\mathrm{B} 2 \& \mathrm{hl}=\mathrm{en} \& \mathrm{sa}=\mathrm{X} \& \mathrm{ei}=\mathrm{VwbtUamLKcv} 7 \mathrm{rAeXr} 4 \mathrm{G} 4 \mathrm{DA} \& \mathrm{ved}=0 \mathrm{CDYQ}$ 6AEwAA

[10] INFORMATION COMMUNICATION SYSTEM AND VEHICLE PORTABLE DEVICE, by Inaguma, Takahiro, Takizawa, Koji, Nishidai, Tetsuo (2013, June 27). Patent US 20130162421 A1 [Online] Available:http://patents.com/us-20130162421.html

[11] Mobile Application for Providing Vehicle Information to Users, by Ross, Steven J. (2013, February 21). Patent US 20130046592 A1 [Online]

Available: http://www.patentstorm.us/applications/20130046592.html

[12] Card reader device for a cell phone and method of use, by Robert E. Morley, Jr. (2010, Oct 12). US 7810729 B2 [Online]. Available: http://www.google.com/patents/US7810729

[13] John G. Proakis, Dmitris G. Manolakis, "Frequnecy Analysis of Signals", in Digital Signal Processing, $4^{\text {th }}$ Edition, Pearson Education, India

[14] Abdul J. Jerri, "The Shannon Sampling Theorem - Its Various Extensions and Applications: A Turtorial Review", Proceedings of the IEEE, Vol. 65, No. 1, November 1977.

[15] Cooley Tukey - J.W. Cooley and J. W. Tukey, "An Algorithm for Machine Computation of Complex Fourier Series", Math. Comp., vol. 19, pp. 297-301, April 1965

[16] MSP 430 - Texas Instruments, MSP430x4xx Family User's Guide (Rev. $J)$, http://www.ti.com/lit/ug/slau056j/slau056j.pdf

[17] GP2YOAO2YK-SHARP, http://sharpworld.com/products/device/lineup/data/pdf/datasheet/gp2y0a 02_e.pdf

[18] Android Developers, http://www.developer.android.com 


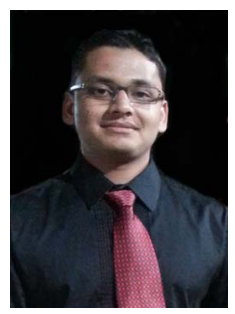

Ajinkya Bari was born in Pune, India in 1992. He is pursuing his Bachelor of Technology (B. Tech) degree in electronics and telecommunication engineering as a senior year student in College of Engineering, Pune and will graduate in 2014.

In May-June 2013, he worked as a Summer Intern at Mahindra \& Mahindra Ltd. Currently he is working as an In-House Project Intern at Tech Mahindra, Pune. He has published a paper titled 'Wireless Gesture Controlled Surveillance Robot' in the proceedings of the ' $7^{\text {th }}$ International Conference on Advanced Computing and Communication Technologies (ICACCT), 2013', vol. 4, pp. 101-105. His current research interests include embedded systems design, parallel computing, computer architecture and robotics.

Mr. Bari is a student member of the Institution of Engineering \& Technology (IET).

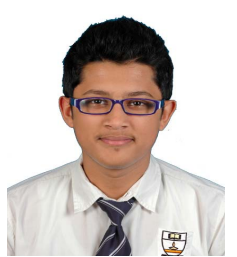

Shraddhesh Bhandari was born in Mumbai, India in 1993. He will receive the B. Tech degree in electronics \& telecommunication engineering from College of Engineering, Pune in 2014. He is currently a final year student at the institution.

He worked as a Summer Intern at Mahindra \& Mahindra Ltd. in 2013. He is currently working as an In-House Project Intern at Tech Mahindra, Pune. His research interests include embedded systems, computer architecture and parallel computing.

Mr. Bhandari is a student member of the Institution of Engineers \& Technology (IET).

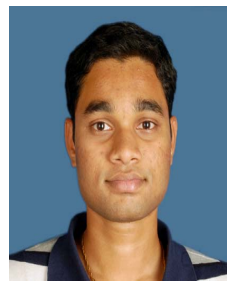

Rajesh Krishnan was born in Mumbai, India in 1992. He received his Diploma in Instrumentation engineering from Vivekanand Education Society's Polytechnic, Mumbai in 2011. He is currently pursuing his B. Tech degree in instrumentation and control engineering from College of Engineering, Pune and will graduate in the year 2014 .

He worked as a summer intern at Mahindra \& Mahindra Ltd. in 2013 and as summer intern at Indian Oil Corporation Ltd. in 2012. His current research interests include reconfigurable computing, embedded systems and computer architecture.

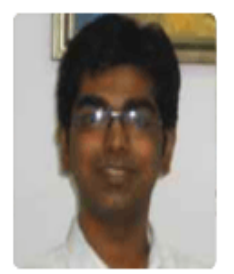

Rohit Pandharkar was born in Pune, India in 1987. He received his B. Tech degree in electronics and telecommunication engineering from College of Engineering, Pune in 2009 and received his Master's degree in Media, Arts and Sciences from Massachusetts Institute of Technology in the year 2011.

Currently he is a Manager, Business Development at Mahindra \& Mahindra Ltd. in Mumbai. He was the Deputy CTO at CanvasM (Mahindra \& Motorola JV) from July 2011- December 2012. He worked as a Graduate Research Assistant at MIT Media Lab from July 2009- June 2011. He was the VP External Affairs at MIT Media Lab India Initiatives from June 2009-2011. In May-July 2008, he worked as a summer intern at India Institute of Technology, Bombay. His subjects of research interest include signal processing, digital image processing, computer vision and optimizations. 